Highest expectations were scored for pain relief and improvement of the ability to walk of short and medium distances. Patients had the lowest expectations for improvement in kneeling, squatting, psychological wellbeing sexual activity and the ability to have paid work.

Female sex, higher age, higher depression score and duration of complaints $>50$ months showed to be significant predictors of lower expectations for the treatment outcome after TKA. Baseline pain and function scores were not related to the level of pre-operative expectations.

Conclusion: In conclusion young, male patients with a short duration of complaints might be at risk of having too high expectations of the treatment result. On the contrary patients with depressive symptoms are more likely to have low expectations, with a potential negative influence on their treatment result. The present study aids in identifying patients at risk for having either too high or too low expectations. This knowledge can be utilized in individualized expectation management interventions.

Acknowledgement: We would like to thank $\mathrm{C}$. van Doesburg and $\mathrm{H}$. Kox for their support in data collection and study procedures.

Disclosure of Interests: None declared

DOI: 10.1136/annrheumdis-2019-eular.5123

\section{THU0464 PHASE 2 CLINICAL TRIAL OF THE GI SAFETY OF A HYDROGEN SULFIDE-RELEASING ANTI- INFLAMMATORY DRUG (ATB-346)}

John Wallace ${ }^{1}$, Andre Buret ${ }^{1}$, Peter Nagy ${ }^{2}$, Marcelo Muscara ${ }^{3}$, Gilberto de Nucci ${ }^{4}$. ${ }^{1}$ University of Calgary, Calgary, Canada; ${ }^{2}$ National Institute of Oncology, Budapest, Hungary; ${ }^{3}$ University of Sao Paulo, Sao Paulo, Brazil; ${ }^{4}$ University of Campinas, Campinas, Brazil

Background: Hydrogen sulfide $\left(\mathrm{H}_{2} \mathrm{~S}\right)$ is a naturally occurring gaseous mediator produced by intestinal bacteria and various eukaryotic cells. $\mathrm{H}_{2} \mathrm{~S}$ exerts anti-inflammatory, pro-resolution and cytoprotective effects in vivo. ATB-346 is an $\mathrm{H}_{2} \mathrm{~S}$-releasing derivative of naproxen, which in animals was shown to produce negligible gastrointestinal (GI) damage and bleeding. In human studies, ATB-346 was found to be much more potent and long-lasting than naproxen. A phase 2 open-label efficacy study demonstrated that ATB-346 (250 mg daily) significantly reduced pain in patients with osteoarthritis of the knee, and markedly suppressed cyclooxygenase (COX) activity. The aim of the present study was to determine if ATB346 would induce less gastroduodenal ulceration than standard dose naproxen.

Objectives: To determine if healthy subjects taking ATB-346 for 14 days would develop significantly less gastroduodenal ulcers $(\geq 3 \mathrm{~mm}$ diameter with depth) than subjects taking an equi-effective dose of naproxen

Methods: This was a double-blind, active control, endoscopic study. 244 healthy volunteers completed the study. Upper Gl endoscopy was performed prior to and on day 14 after commencing treatment with naproxen (550 mg twice daily) or ATB-346 (250 mg) once daily in the morning and placebo once daily in the evening. Whole blood thromboxane synthesis was measured on days 0,7 and 14 . Plasma $\mathrm{H}_{2} \mathrm{~S}$ levels were also measured.

Results: 53 subjects taking naproxen (42.2\%) developed at least one ulcer, while only 3 subjects (2.5\%) treated with ATB-346 developed at least one ulcer $(\mathrm{p}<0.0001)$. The two drugs suppressed COX activity to the same extent $(>95 \%)$. Affected subjects in the naproxen group developed more ulcers (an average of 4 per subject) than in the ATB-346 group (an average of 1.3), and there was a much greater incidence of larger ulcers $(\geq 5 \mathrm{~mm}$ diameter) in the naproxen group than in the ATB346 group (125 vs 0 , respectively). The incidence of gastro-esophageal reflux, abdominal pain and nausea was lower with ATB-346 than with naproxen. Plasma $\mathrm{H}_{2} \mathrm{~S}$ levels were significantly elevated (by $50 \%$; $p<0.001)$ in the ATB-346 group.

Conclusion: Consistent with the pre-clinical studies, this phase 2 clinical trial demonstrated a dramatic reduction of upper Gl ulcer formation in subjects treated with equi-effective doses of ATB-346 versus naproxen. The COX inhibition observed in this trial was consistent with a previous phase $2 \mathrm{~A}$ trial that demonstrated significant pain relief with ATB-346 in patients with osteoarthritis of the knee. ATB-346 appears to be an effective and much safer alternative to existing NSAIDs.

Disclosure of Interests: : John Wallace: None declared, Andre Buret Shareholder of: Antibe Therapeutics, Peter Nagy: None declared, Marcelo Muscara Grant/research support from: Antibe Therapeutics, Gilberto de Nucci Shareholder of: Antibe Therapeutics, Employee of: BioLab Brasil DOI: 10.1136/annrheumdis-2019-eular.568

\section{THU0464B IS THERE AN ASSOCIATION BETWEEN METABOLIC SYNDROME AND SEVERITY OF HAND OSTEOARTHRITIS? RESULTS FROM A NATIONWIDE STUDY}

Margarida Cruz ${ }^{1}$, Alexandre Sepriano ${ }^{2}$, Sara Dias ${ }^{3}$, Ana Maria Rodrigues ${ }^{4}$ Helena Canhão ${ }^{3}$, Nélia Gouveia ${ }^{2}$, Mónica Eusébio ${ }^{5}$, Sofia Ramiro ${ }^{6}$, Jaime Branco ${ }^{7}{ }^{1}$ Nova Medical School - Lisbon, Lisbon, Portugal; ${ }^{2}$ Faculdade de Ciências Médicas, CEDOC, Lisboa, Portugal, ${ }^{3}$ Faculdade de Ciências Médicas, CEDOC, EpiDoc, Lisboa, Portugal; ${ }^{4}$ Faculdade de Ciências Médicas, Lisboa, Portugal; ${ }^{5}$ Sociedade Portuguesa de Reumatologia, Lisboa, Portugal; ${ }^{6}$ Leiden University Medical Center, Leiden, Netherlands; ${ }^{7}$ Nova Medical School, CEDOC, Lisboa, Portugal

Background: Hand osteoarthritis ( $\mathrm{HOA})$ is a highly prevalent rheumatic disease that predominates in females and causes pain, joint deformities and loss of functional capacity. Overweight and metabolic syndrome have been previously suggested to associate with the severity of HOA, but clarity on these associations is yet to be achieved.

Objectives: To test the possible association between body mass index (BMI) and other individual components of metabolic syndrome with severity of $\mathrm{HOA}$ in females from a nationwide epidemiological study.

Methods: EpiReumaPt was a three-stage national health survey where, in the first phase, 10,661 adult participants were randomly selected and interviewed using a structured face-to-face questionnaire that included screening for rheumatic diseases, such as HOA. In the second phase, positive screenings for $\geq 1$ rheumatic complaint plus $20 \%$ of the negative screenings were invited for an assessment by rheumatologists. Finally, 3 rheumatologists revised all the information and defined the final diagnosis by consensus. Female patients with a final clinical diagnosis of primary HOA were included in this analysis. Hand functional status as assed by the Cochin questionnaire was the outcome of interest. The explanatory variables of interest were: BMI evaluated as a categorical variable (Normal: 18-24.99; overweight: 25-29.99; obesity: $\geq 30$ ), diabetes mellitus, hypertension and hypercholesterolemia (all self-reported and as binary variables: yes/no). The possible associations between BMI and the individual components of the metabolic syndrome with the Cochin score were tested in a multivariable linear regression model. Only significant variables $(p<0,05)$ were kept in the final model. Potential confounders of the associations of interest and the outcome were defined a priori on clinical grounds and included age and symptoms of depression (HADS score).

Results: Out of the 3,877 participants evaluated by Rheumatologists, 473 women had primary HOA (national prevalence: $6.6 \%$ ). In this population, $40 \%$ were overweight and $29 \%$ were obese. Ninety-three $(20 \%)$ participants had diabetes, 261 (56\%) had hypertension and 261 (56\%) had hypercholesterolemia. In the multiple regression model, BMI and diabetes were found to significantly associate with HOA severity, whereas hypertension and hypercholesterolemia did not, thus not being selected in the final model (table).

Abstract THU0464 -Table 1. Association between individual components of the metabolic syndrome and HOA severity (Cochin score). Multivariable linear regression model

\begin{tabular}{lc} 
& HOA severity (Cochin score) \\
$\beta$ coefficient $(95 \% \mathrm{Cl})$ & $\mathrm{N}=408$ \\
\hline BMl (categorical) & $0.31(0.05 ; 0.57)$ \\
Diabetes (yes vs no) & $3.63(0.13 ; 7.13)$ \\
Age (years) & $0.13(-0.01 ; 0.27)$ \\
HADS score (continuous) & $0.90(0.59 ; 1.22)$ \\
\hline
\end{tabular}

Conclusion: In this study, higher BMI and the presence of diabetes mellitus associated with a worse functional capacity in women with primary HOA. These data add to the body of evidence suggesting a possible role of metabolic factors in the severity of HOA.

\section{REFERENCES:}

[1] Yusuf

E, Nelissen RG, loan-Facsinay A et al. Association between weight or body mass index and hand osteoarthritis: a systematic review. Ann Rheum Dis 2010. 69: 761-765

[2] Schett G, Kleyer A, Perricone $C$ et al. Diabetes is an independent predictor for severe osteoarthritis: results from a longitudinal cohort study. Diabetes Care 2013; 36: 403-409

Acknowledgement: The authors thank all the participants of EpiReumaPt survey, the interviewers and staff and the Rheumatologists that worked in the field Disclosure of Interests: Margarida Cruz: None declared, Alexandre Sepriano: None declared, Sara Dias: None declared, Ana Maria 
Rodrigues: None declared, Helena Canhão: None declared, Nélia Gouveia: None declared, Mónica Eusébio: None declared, Sofia Ramiro Grant/research support from: MSD, Consultant for: AbbVie, Lilly, MSD, Novartis, Pfizer, Sanofi, Speakers bureau: AbbVie, Lilly, MSD, Novartis, Pfizer, Sanofi, Jaime Branco: None declared DOI: 10.1136/annrheumdis-2019-eular.6400

\section{THU0465 PHARMACOKINETICS AND TOXICOKINETICS STUDIES OF A SUSTAINED RELEASE LIPOSOMAL FORMULATION OF DEXAMETHASONE SODIUM PHOSPHATE (TLC599) FOLLOWING INTRA-ARTICULAR INJECTION IN DOGS}

Tzung-Ju Wu, Wan-Ni Yu, Ming-Ju Wu, Po-Chun Chang, Sheue-Fang Shih. Taiwan Liposome Company, Taipei, Taiwan, Republic of China

Background: Osteoarthritis $(\mathrm{OA})$ is a degenerative joint disorder with limited long-lasting treatment options. Various steroid formulations on the market are effective but required frequent intra-articular (IA) injections due to short-term symptomatic relief. IA steroids have been shown to increase cartilage loss in knee and the rapid absorption into systemic circulation could cause adverse side effects, limiting their effectiveness [1]. To circumvent these issues, TLC599, a novel sustained-release liposome formulation of dexamethasone sodium phosphate (DSP) was developed to provide a sustained $O A$ pain management over an extended period with reduced side effects and toxicity. TLC599 resulted in sustained presence in the synovial fluid without significant local and systemic side effects from $O A$ treatments following IA injection.

Objectives: To evaluate pharmacokinetics (PK) and toxicokinetics (TK) of TLC599 following IA injection in dogs.

Methods: In two studies, the dexamethasone phosphate (DP) and dexamethasone (DEX) concentration was quantified and the PK/TK profile of TLC599 was evaluated in dogs following IA injection. DSP is the sodium salt form of DP and DEX is the active form of DSP. Study\#8351851: TLC599 was administered as a single- or multiple-dose injection (once

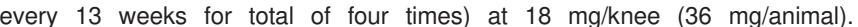
Synovial fluid was collected at 2.5, 48, 96, 168 and 360 hours post-dose from 4 animals/time point, and plasma was collected at $0.25,0.75,1.25$, 2, 4, 6, 24, 48, 96 and 168 hours post-dose from 8 animals/time point. Study\#8388198: TLC599 was administered as a single-dose injection at $18 \mathrm{mg} / \mathrm{knee}$. The synovial fluid was collected at 15, 30, 45, 90, and 120 days post-dose from 4 animals/time point.

Results: Study\#8351851: Following a single-dose IA injection of TLC599, DP concentration declined gradually after $2.5 \mathrm{hr}$ but remained detectable through $360 \mathrm{hr}$, demonstrating the prolonged local exposure in the joint (Figure 1). The PK parameters of DP and DEX in dog plasma were assessed in the multiple-dose group. The mean $C_{\max }$ and $A \cup C_{0-t}$ values of DP and DEX were comparable between the first dose (Day 1) and the fourth dose (Day 274) (Table 1), indicating there was no accumulation of systemic exposure of DP and DEX following four repeated IA injections of TLC599. By evaluating the effect on cartilage, it was also shown that the local safety profiles were similar between multiple-dose and single-dose TLC599 injections.

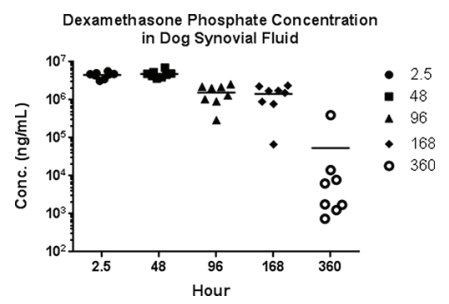

Abstract THU465 - Figure 1. Mean Concentration of DP in Dog Synovial Fluid.

Abstract THU465 - Table 1. Summary of Mean $\mathrm{C}_{\max }$ and $\mathrm{AUC}_{0-\mathrm{t}}$ of DP and DEX in Dog Plasma.

\begin{tabular}{|c|c|c|c|c|}
\hline Analyte & Dose\# & $\begin{array}{l}\text { Dose Level } \\
\text { (mg/animal) }\end{array}$ & $\begin{array}{c}\mathrm{C}_{\max } \\
(\mathrm{ng} g \mathrm{~mL})\end{array}$ & $\begin{array}{c}\text { AUC } C_{0-1} \\
\text { hour }{ }^{*} n g / m \text { ) }\end{array}$ \\
\hline \multirow{2}{*}{ Dexamethasone Phosphate } & $\begin{array}{l}\text { First Dose } \\
\text { (Day 1) }\end{array}$ & 36 & 450 & 419 \\
\hline & $\begin{array}{c}\text { Fourth Dose } \\
\text { (Day 274) }\end{array}$ & 36 & 522 & 505 \\
\hline \multirow{2}{*}{ Dexamethasone } & $\begin{array}{l}\text { First Dose } \\
\text { (Day 1) }\end{array}$ & 36 & 498 & 6385 \\
\hline & $\begin{array}{l}\text { Fourth Dose } \\
\text { (Day 274) }\end{array}$ & 36 & 427 & 5955 \\
\hline
\end{tabular}

Study\#8388198: Following a single-dose IA injection of TLC599, DP concentration declined after 15 days but remained at similar level from 30 days to 120 days post-dose. Overall, the prolonged 120-day residence time of TLC599 in synovial joint was observed (Figure 2).

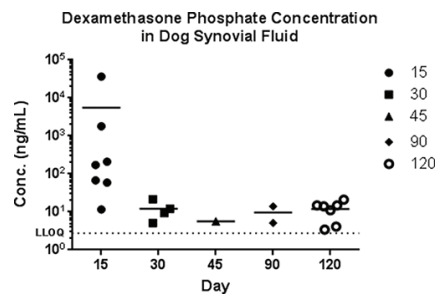

Abstract THU465 - Figure 2. Mean Concentration of DP in Dog Synovial Fluid.

Conclusion: TLC599, a novel extended-release liposome formulation of DSP, showed a long-lasting profile up to 120 days in synovial joint after a single IA injection in a preclinical dog study. In addition, no significant systemic exposure and accumulation of DP and DEX in dog plasma was observed following multiple-dose administration of TLC599. Animal studies indicate that TLC599 may be an effective and superior chronic treatment for $O A$.

\section{REFERENCE:}

[1] Zhang W, et al., OARSI recommendations for the management of hip and knee osteoarthritis, part II: OARSI evidence-based, expert consensus guidelines. Osteoarthritis Cartilage 2008;16(2): 137-62

Acknowledgement: The authors thank Yingfang Li, Jiunmin Lai, Ruixue Chen, Ph.D. and Carl Brown Ph.D. for reviewing support.

Disclosure of Interests: Tzung-Ju Wu Employee of: Current employee of Taiwan Liposome Company, Wan-Ni Yu Employee of: Current employee of Taiwan Liposome Company, Ming-Ju Wu Employee of: Current employee of Taiwan Liposome Company, Po-Chun Chang Employee of: Current employee of Taiwan Liposome Company, Sheue-Fang Shih Employee of: Current employee of Taiwan Liposome Company DOI: 10.1136/annrheumdis-2019-eular.3880

\section{THU0466 THE EVALUATION OF THE EFFECTIVENESS OF INTRA- ARTICULAR STEROID, TENOXICAM AND COMBINED STEROID-TENOXICAM INJECTIONS IN THE TREATMENT OF PATIENTS WITH KNEE OSTEOARTHRITIS}

YILMAZ Ebru . Korfez Government Hospital, Physical Therapy and Rehabilitation, Kocaeli, Turkey

Background: Intra-articular corticosteroid injections are widely applied in the treatment of symptomatic knee osteoarthritis $(\mathrm{OA})$. There is an evidence of short-term effects of intra-articular corticosteroid injection (up to 3-4 weeks), however there is no consensus for the long-term benefit of this treatment yet (1). Tenoxicam is an effective analgesic and anti-inflammatory drug for symptomatic treatment of OA. Additionally, apart from oral use, tenoxicam is also applied as an intra-articular treatment option to minimize gastrointestinal side effects of NSAIDs (2). Clinical evidence suggests that the combined use of NSAIDs and corticosteroids is synergistic (especially macular edema after cataract surgery in ophthalmology) (3)

Objectives: The aim of this study is to compare the effectiveness of intra-articular administration of these treatments and their combination and determine whether the combination of intraarticular steroid and tenoxicam was more effective for a long period rather than only tenoxicam and steroid injection alone in $\mathrm{OA}$ treatment.

Methods: 90 patients (56 female, 34 male) with diagnosis of knee osteoarthritis were randomly divided into three groups (30 patients per group): Group 1 were treated by intra-articular injection of tenoxicam. Group 2 were treated by intra-articular injection of triamcinolone hexacetonide. Group 3 were treated by intra-articular injection of triamcinolone hexacetonide combined with tenoxicam. The estimation of the severity of pain by the visual analog scale (VAS) were enrolled at baseline and 1, 3, 6 months post-injection. Additionally, the Western Ontario and McMaster Universities Index (WOMAC) was used to determine the outcome measures of pain, stiffness and physical functioning at baseline and 1, 3, 6 months post-injection. 
Results: The mean age of patients was $65.97 \pm 9.29$ years. In tenoxicam group, median pre- and post-treatment (at 1,3 and 6 months) VAS/ WOMAC scores were 7.00/32.00, 2.00/10.00, 7.00/32.00 and 7.00/32.00, respectively. In steroid group, median pre- and post-treatment (at 1, 3 and 6 months) VAS/WOMAC scores were 8.00/34.00, 1.00/8.00, 8.00/ 34.00 and $8.00 / 34.00$, respectively. In steroid plus tenoxicam group, median pre- and post-treatment (at 1,3 and 6 months) VAS/WOMAC scores were $7.00 / 34.00,0.00 / 6.00,1.00 / 8.00$ and $2.00 / 10.00$, respectively. VAS and WOMAC scores in 1 month after the injection significantly decreased in both groups compared to baseline $(p<0.01)$. However, there was a pronounced improvement in only steroid plus tenoxicam group at 3 and 6 months post-injection $(\mathrm{p}<0.01)$. Steroid plus tenoxicam group showed significantly improved VAS and WOMAC scores when compared to only steroid and tenoxicam group at follow-up 3 and 6 months $(p<0.01)$

\section{Abstract THU0466 -Table 1.}

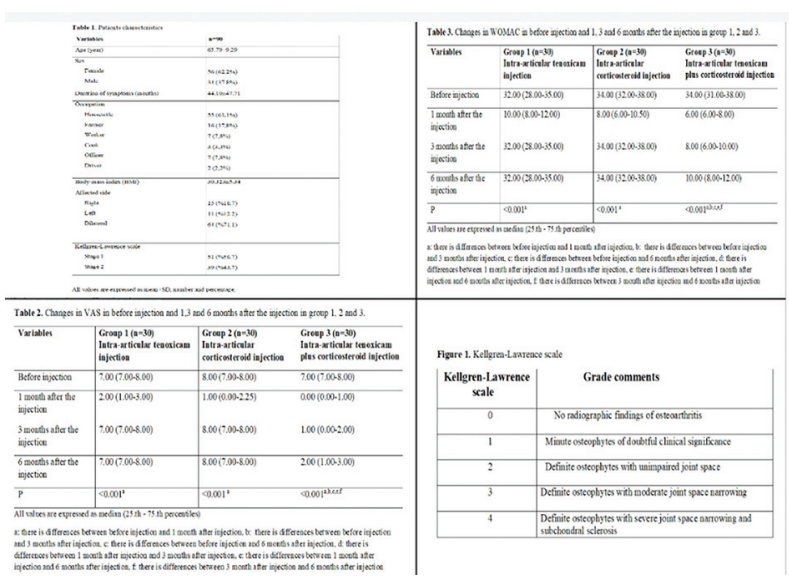

Abstract THU0466 -Table 2.

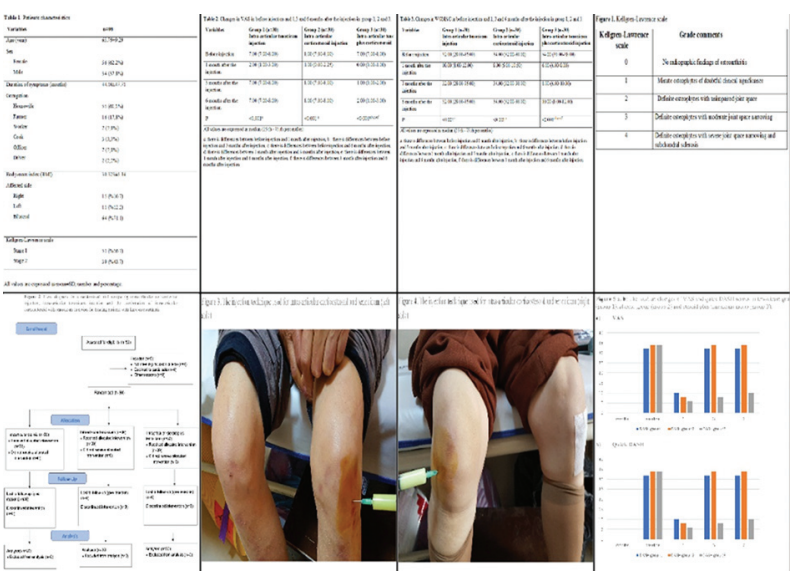

Conclusion: The combination of corticosteroids and tenoxicam seems to produce a more effective result than alone therapy in reducing pain and improving functional recovery.

\section{REFERENCES:}

1) Maricar N, Callaghan MJ, Felson DT, O'Neill TW. Predictors of response to intra-articular steroid injections in knee osteoarthritis- a systematic review. Rheumatology. 2013;52:1022-1032.

[2] Evcik D, Kızılay B, Maralcan G. The efficacy of intraarticular tenoxicam in the treatment of knee osteoarthritis. The Pain Clinic. 2003;15:405-408.

[3] Malik A, Sadafale A, Gupta YK, Gupta A. A comparative study of various topical nonsteroidal anti-inflammatory drugs to steroid drops for control of post cataract surgery inflammation. Oman J Ophthalmol. 2016;9:150-156.

Acknowledgement: None

Disclosure of Interests: None declared

DOI: 10.1136/annrheumdis-2019-eular.265

\section{THU0467 \\ DOES GENERALISED PAIN AND LOCALISED PAIN SEVERITY INCREASE RISK OF PREVALENT AND INCIDENT FRACTURES IN OLDER ADULTS?}

Feng Pan ${ }^{1}$, Jing Tian ${ }^{1}$, Dawn Aitken ${ }^{1}$, Flavia Cicuttini' ${ }^{2}$, Graeme Jones ${ }^{1} .{ }^{1}$ University of Tasmania, Menzies Institute for Medical Research, Hobart, Australia; ${ }^{2}$ Monash University Medical School, Department of Epidemiology and Preventive Medicine, Melbourne, Australia

Background: Musculoskeletal pain is common particularly in the elderly and typically occurs in multiple sites. Pain has been linked to falls risk however, whether increased risk of falls related to pain can subsequently increase fracture risks remains unclear.

Objectives: To describe the associations of localised pain severity and generalised pain (number of painful sites) with incident fractures, and to explore whether their associations are independent of falls risk, bone mineral density (BMD) and potential confounders.

Methods: Data from a longitudinal population-based study of older adults (mean age 63 years, 51\% female) were utilised. A mean follow-up was performed at 2.6, 5.1 and 10.7 years later, respectively. Pain severity in the knee was measured by the Western Ontario and McMaster Universities Osteoarthritis Index pain questionnaire. Presence/absence of pain at the neck, back, hands, shoulders, hips, knees and feet was assessed by questionnaire at baseline. Fractures were self-reported at each time-point. BMD was measured by Dual-energy X-ray absorptiometry (DXA). Falls risk was calculated based on the short form Physiological Profile Assessment.

Results: A total of 455 fractures at baseline and 154 new fractures were reported during follow-up. In multivariable analyses, both pain severity and number of painful sites were associated with prevalent fractures at any site. Pain severity was associated with prevalent vertebral fractures, while number of painful sites was associated with prevalent fractures at non-vertebral and hip sites. Furthermore, pain severity was associated with an increased risk of incident fractures at any site [relative risk (RR) $1.04,95 \% \mathrm{Cl} 1.02-1.06$ ], major (including the femur, radius, ulnar, vertebral, rib and humerus) (RR $1.10,95 \% \mathrm{Cl} 1.05-1.15)$ and vertebral (RR $1.04,95 \% \mathrm{Cl} 1.01-1.08)$. Similarly, number of painful sites was also associated with increased risk of incident fractures at any site [RR 1.69, 95\% $\mathrm{Cl}$ 1.13-2.53], major ( $\mathrm{RR} 2.17,95 \% \mathrm{Cl} 1.12-4.22$ ) and vertebral (RR 6.44 $95 \% \mathrm{Cl}$ 1.64-25.33). There was a dose-response relationship between number of painful sites and risk of incident fractures. These associations remained significant after further adjustment for falls risk and BMD. No significant associations were found for fractures occurring at non-vertebral and hip sites.

Conclusion: Both pain severity and generalised pain are associated with increased risk of prevalent and incident fractures, which is independent of falls risk, BMD and potential confounders, suggesting that pain may be an independent marker of fracture risk. Improved pain management may have the potential to prevent fractures in older adults.

Disclosure of Interests: : None declared

DOI: 10.1136/annrheumdis-2019-eular.4040

\section{Fibromyalgia}

\section{THU0468 THE INTERACTIONS OF PHYSICAL ACTIVITY LEVELS WITH THE SODIUM CHANNEL PROTEIN TYPE 9 SUBUNIT ALPHA AND METHYLENE TETRAHYDROFOLATE REDUCTASE GENES ARE ASSOCIATED WITH FATIGUE IN WOMEN WITH FIBROMYALGIA}

Fernando Estevez-Lopez ${ }^{1,2,3}$, Diego Salazar-Tortosa ${ }^{2}$, Blanca Gavilán Carrera ${ }^{2}$, Virginia A Aparicio ${ }^{2}$, Pedro Acosta-Manzano ${ }^{2}$, Víctor Segura-Jiménez ${ }^{4}$, Inmaculada C Alvarez-Gallardo ${ }^{4}$, Ana Carbonell-Baeza ${ }^{4}$, Diego MunguíaIzquierdo ${ }^{5}$, Rinie Geenen 1 , Eliana Lacerda ${ }^{6}$, Manuel Delgado-Fernández ${ }^{2}$, Luis J Martínez-González ${ }^{7}$, Jonatan R Ruiz ${ }^{2}$, María J Álvarez-Cubero ${ }^{2,7}$. ${ }^{1}$ Utrecht University, Utrecht, Netherlands; ${ }^{2}$ University of Granada, Granada, Spain; ${ }^{3}$ Ulster University, Belfast, United Kingdom; ${ }^{4}$ University of Cádiz, Cádiz, Spain;

${ }^{5}$ Universidad Pablo de Olavide, Seville, Spain; ${ }^{6}$ The London School of Hygiene and Tropical Medicine, London, Spain; ${ }^{7}$ Liquid biopsy and metastasis research group and Genomic Unit, GENyO (Pfizer-University of Granada-Andalusian Government Centre for Genomics and Oncological Research), Granada, Spain

Background: People with fibromyalgia identify fatigue as one of the main symptoms of the disease [1]. It is hypothesised that the pathogenesis of fibromyalgia involves a genetic susceptibility that is modulated by environmental factors [2] 